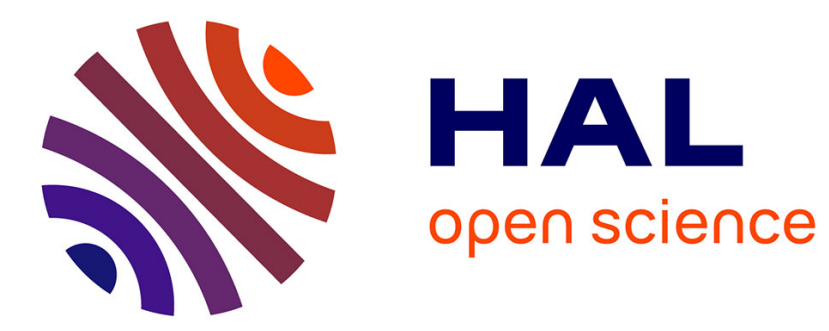

\title{
Los fondos de Lidia Falcón: un archivo para la historia del movimiento feminista desde los 60
}

Claudia Jareño

\section{To cite this version:}

Claudia Jareño. Los fondos de Lidia Falcón: un archivo para la historia del movimiento feminista desde los 60. Investigaciones Feministas, inPress, 10 (1), pp.27-44. 10.5209/INFE.60722 . hal-03140611

\section{HAL Id: hal-03140611 \\ https://hal.science/hal-03140611}

Submitted on 13 Feb 2021

HAL is a multi-disciplinary open access archive for the deposit and dissemination of scientific research documents, whether they are published or not. The documents may come from teaching and research institutions in France or abroad, or from public or private research centers.
L'archive ouverte pluridisciplinaire HAL, est destinée au dépôt et à la diffusion de documents scientifiques de niveau recherche, publiés ou non, émanant des établissements d'enseignement et de recherche français ou étrangers, des laboratoires publics ou privés. 


\title{
Investigaciones Feministas
}

ISSN: 1131-8635

\section{Los fondos de Lidia Falcón: un archivo para la historia del movimiento feminista desde los 60}

\author{
Claudia Jareño Gila
}

Recibido: Junio 2018 / Revisado: Abril 2019/ Aceptado: Mayo 2019

Resumen. Uno de los mayores retos de la historia de las luchas por los derechos de las mujeres es la recuperación y la conservación de los documentos generados por las asociaciones, colectivos e individualidades que conformaron el Movimiento Feminista durante la transición. En este artículo, con el objetivo de dar a conocer una parte de su documentación, se presentan los fondos de Lidia Falcón, así como del Partido Feminista y el Club Vindicación Feminista, depositados en el Archivo Nacional de Cataluña. Este conjunto alberga toda la documentación gestada en los múltiples proyectos fomentados por Falcón -abogada, escritora, periodista, pero también dramaturga e infatigable militante feminista-, entre los que destaca la publicación de dos revistas: Vindicación Feminista y Poder y Libertad, así como la fundación del Partido Feminista de España. En este sentido, la diversidad y el volumen de la documentación hacen de estos fondos unos de los más importantes para el estudio y la reconstrucción de la historia del movimiento feminista contemporáneo en el Estado español.

Palabras clave: Archivos, feminismo, Vindicación Feminista, Lidia Falcón.

\section{[en] The collection of Lidia Falcón: an archive for the history of the feminist movement since the 1960 s}

\begin{abstract}
One of the greatest challenges in the history of struggles for women's rights is the collection and conservation of documents generated by associations, collectives and individuals that were part of the Feminist Movement during the Spanish Transition to democracy. With the aim of highlighting part of their content, this article presents the Lidia Falcón Fonds as well as the Feminist Party and the Feminist Vindication Club Fonds, available for consultation at the National Archive of Catalonia. The fonds consist of all the documents produced in many projects led by Falcón -a lawyer, writer, journalist but also a dramatist and untiring feminist militant- among which we may underline the publication of two magazines -Vindicación Feminista y Poder y Libertad - as well as the foundation of the Feminist Party of Spain. Therefore, the diversity and volume of documents collected make these two fonds essential for the study and reconstruction of the history of this movement.
\end{abstract}

Keywords: Archives, feminism, Feminist Vindication, Lidia Falcón.

Sumario. 1. Introducción. 2. Un repaso por los archivos y bibliotecas feministas del Estado español. 3. Los fondos feministas del Archivo Nacional de Catalunya: el fondo Lidia Falcón y el fondo Partido Feminista-Club Vindicación Feminista. 3.1. El fondo Lidia Falcón: una genealogía feminista. 3.2. El fondo Partido Feminista de España - Club Vindicación Feminista: la historia de una larga lucha. 3.3. Correspondencia, colaboraciones y amistad. 4. Conclusiones. Agradecimientos. Referencias bibliográficas.

1 Universidad de París 8. Francia.

claudia.jareno@univ-rouen.fr 
Cómo citar: Jareño Gila, C. (2019). Los fondos de Lidia Falcón: un archivo para la historia del movimiento feminista desde los 60, en Investigaciones feministas 10.1, 133-147.

\section{Introducción}

En el prólogo del libro de Carmen Alcalde, La mujer en la Guerra civil española, publicado en 1976, Lidia Falcón afirmaba que uno de los mayores desafíos con los que se había topado la escritora y periodista catalana para la elaboración de la obra había sido «encontrar en los archivos mutilados de las hemerotecas, el testimonio inmediato de la historia de nuestras mujeres [...]. Rebuscar en las librerías de viejo los olvidados libros, los menospreciados panfletos que trataron un día cualquier reivindicación femenina» $(1976,15)$. Veinte años más tarde, en otro libro de la misma autora, Mujeres en el Franquismo (1996), Carmen Alcalde volvía de nuevo a señalar las dificultades a las que se había tenido que enfrentar en su investigación, rebuscando y hurgando en archivos, hemerotecas y archivos privados para encontrar las huellas dejadas por las mujeres:

¿Y las mujeres? ¿Existieron las mujeres? En la memoria de los hombres que han relatado la historia, parece que no. Ni las que pudieron huir ni las que se quedaron constan en ningún, en ningún índice onomástico de los centenares de libros escritos sobre la guerra civil, el exilio y la resistencia. Lo confirmo con certeza de no equivocarme. He pasado largas horas en este investigación para recoger datos para esta historia de las mujeres y, aun queriendo encontrarlas con lupa, a pesar de saber que haberlas hubo, «y tantas! no parece que las "hayla"»(1996, 88-89).

Y es que, como ya advirtiera también Adrienne Rich (2011) en Sobre mentiras, secretos y silencios, uno de los mayores retos de la historia de la lucha por la autodeterminación de las mujeres ha sido el intento recurrente de ocultarla una y otra vez, junto con las dificultades para conservar, organizar, catalogar y almacenar las cantidades ingentes de documentación que generaron los colectivos y militantes feministas a lo largo de los diferentes momentos de su activismo político.

Una de las primeras cuestiones que debemos abordar a la hora de estudiar las huellas materiales producidas por los colectivos feministas es saber a qué nos referimos cuando hablamos de fondo feminista. Siguiendo la definición acuñada por Christine Bard, historiadora sobre el feminismo y una de las fundadoras del Archive du féminisme en Francia, entendemos por fondo feminista todo el conjunto de documentos originales (papeles, manuscritos, correspondencia...) elaborada por asociaciones o personalidades feministas en el marco de sus actividades o recogidas por coleccionistas (2006, 23). Otras autoras prefieren hablar de archivo feminista, constituido por los documentos de archivo producidos por agentes en sus actividades de lucha por los derechos de las mujeres (Codina y San Segundo, 2016, 4).

Igualmente, son fundamentales los fondos personales de mujeres que, como el de la feminista y abogada Lidia Falcón, estuvieron vinculadas a alguna asociación o grupo feminista. Se trata, como dice Valérie Freschet de una memoria caliente, es decir, de fondos documentales con gran cantidad de correspondencia, cartas, memorias y toda una clase de documentos más cercanos a la denominada escritura del yo, 
en oposición a la memoria fría, aquella memoria generada en las instituciones del poder (Jornet y Tuset, 2005, 2).

Una propuesta complementaria para abordar el tema de los archivos feministas sería la del «archivo de lo difuso», de María Rosón y Rosa Medina Domenech, que incluiría materiales visuales como la fotografía -el artículo presta especial atención a la cultura visual-, que representan resistencias «emocionales» al poder, pero englobaría también canciones, gestos, o palabras que desafían potencialmente las diferentes formas de poder $(2017,420)$. En efecto, aunque esta propuesta no incluya la documentación generada por los colectivos feministas con voluntad explícita de enfrentamiento o de cuestionamiento del orden de género, es sumamente útil para comprender y valorar aquello que no se archiva y que, generalmente, ha sido dejado de lado por los archivos convencionales -aquellos que tienen que ver con la memoria fría.

Asimismo, en otros casos, la recuperación de las memorias de los feminismos ${ }^{2}$ ha pasado por la recogida de testimonios orales de las protagonistas -algunos dentro de proyectos de construcción de archivos orales realizados en el marco de investigaciones universitarias o desde colectivos militantes, dándose, en muchos casos, una estrecha colaboración entre ambos ${ }^{3}$.

\section{Un repaso por los archivos y bibliotecas feministas del Estado español}

Hace más de un siglo que en Europa se empezaron a crear centros de documentación, archivos y bibliotecas de mujeres que, a la par del movimiento feminista, y muchas veces en colaboración, han intentado conservar una memoria de los feminismos. Una memoria material diversa en cuanto a sus soportes tangibles, pues engloba desde artículos y recortes de prensa, actas de jornadas y encuentros significativos para la historia del movimiento de las mujeres, estatutos, reglamentos de organizaciones, publicaciones periódicas (fanzines y revistas), trípticos y folletos, hasta objetos como carteles, pósteres y pancartas o chapas, pero también material audiovisual como vídeos y grabaciones sonoras o fotografías.

En el Estado español, la primera iniciativa remonta a 1909, con la creación en Barcelona, por Francesca Bonnemaison, del Institut de Cultura i Biblioteca Popular per a la Dona, ahora llamada por el nombre de su fundadora, que fue la primera biblioteca europea con un fondo especializado sobre mujeres (Llinàs, 2008, 19) y

2 Según Núria Jornet i Benito y Núria Tuset Páez, por memoria de los feminismos se entiende: «el patrimonio bibliográfico y documental generado por las asociaciones feministas y los grupos de mujeres vinculadas al feminismo, y por las mujeres que participaron directamente o pueden ligarse a este movimiento. También forman parte las bibliotecas y centros de documentación fundados a lo largo de la cronología del movimiento que [...] se pueden vincular a las políticas gubernamentales de igualdad, a las estructuras académicas y de investigación, o en las propias entidades y asociaciones nacidas con el feminismo» (Jornet y Tuset, 2016, 2).

3 Un ejemplo lo encontramos en el proyecto titulado «Mujeres y Transición» dirigido por el grupo de la Universidad de Alicante, «Haciendo Historia: género y transición política en España» (MICINN.FEM 2010-19068), que incluye, entre otras actividades, la realización de más de una veintena de entrevistas a mujeres que vivieron la Transición desde diferentes ámbitos, algunas militantes y otras no, aunque en su inmensa mayoría mujeres de izquierda. Página web del proyecto: http://www.mujeresytransicion.es/pages/home.php

Otro ejemplo es el de «Feministas valencianas», creado por Doleres Sánchez Durá, que sigue el mismo esquema que el grupo anterior, pero que en este caso entrevista únicamente a mujeres feministas que durante la Transición tuvieron una papel muy activo en el movimiento feminista. https://feministasvalencianas.wordpress.com/ 
que posee, además, un valioso fondo sobre obras entre finales del siglo XIX y el comienzo de la Guerra Civil.

Más recientes son las iniciativas surgidas al calor del Movimiento Feminista de los años 70, tras la «travesía del desierto» de la dictadura, en muchos casos promovidas por los propios colectivos feministas. De duración más efímera fueron iniciativas como la de la La Sal, que empezó a funcionar como bar-biblioteca el 6 de junio de 1977 en la calle Riereta, número 8 de Barcelona (Almerini, 2014). Entre sus diversas actividades auto-gestionadas se incluía un centro de información y documentación feminista y una «biblioteca de temas feministas» (Llinàs, 2008, 114-115) con importantes colaboraciones de las editoriales del momento, lo que representó uno de los primeros ensayos de biblioteca feminista que llegó a contar con 400 volúmenes (Robles y Medina, 2000, 60).

También en Barcelona, en la calle Ripoll, se encuentra el Centro de Documentación de la asociación feminista $\mathrm{Ca}$ la Dona, centro feminista creado en enero de 1988 tras intensos debates con el ayuntamiento de Barcelona (Cervera et al., 1997, 11), que guarda una ingente documentación sobre el feminismo catalán, en especial sobre los grupos independientes como LA MAR. El Centro de Documentación Ca la Dona, además de archivo gráfico y administrativo propio, contiene los fondos personales diversas militantes protagonistas del Movimiento Feminista como el de Gretel Amman Martínez ${ }^{4}$, donados tras su desaparición en 2000 por su compañera. El Centro de documentación de Ca la Dona está adscrito, además, al proyecto europeo FRAGEN (FRAmes on GENder) que empezó a gestarse en el 2008, coordinado por el Instituto de Historia de las Mujeres de Ámsterdam Atria (Institute on gender equality and woman's history) ${ }^{5}$, cuyo objetivo es facilitar los estudios comparativos sobre la historia del pensamiento feminista en Europa mediante la creación de una base de datos, con los textos clave para el desarrollo del movimiento feminista en veintinueve países europeos durante la segunda mitad del siglo $\mathrm{XX}^{6}$.

En 1983, la Federación Provincial de Asociaciones de Mujeres «Flora Tristán», ligada al Movimiento Democrático de Mujeres -asociación creada a finales de 1964 a instancias del Partido Comunista y cuyo papel en las movilizaciones vecinales durante el franquismo fue destacado- impulsó la creación del Centro de Información y Formación Feminista (CIFFE), radicado en Madrid y que actualmente se conserva en el Centro Documental de la Memoria Histórica (CDMH) de Salamanca ${ }^{7}$. El Centro buscaba «el trabajo formativo, cultural y de concienciación de las mujeres» mediante una serie de actividades «en tres áreas: seminarios, cultural, documentación y estudios sociológicos» ${ }^{8}$. El área documental tuvo como cometido recopilar todo tipo de materiales relacionados con el Movimiento Feminista y, de forma muy especial, con la historia del feminismo en España ${ }^{9}$. Cabe destacar su archivo sonoro, compuesto por más de doscientas cincuenta entrevistas a destacadas participantes

\footnotetext{
http://www.caladona.org/centredocumentacio/gretel/biografia/biografia.html

https://www.atria.nl/en

http://www.fragen.nu/atria/fragen/

Una primera consulta muy somera sobre este fondo se puede hacer a través de la página web de PARES http:// pares.mcu.es/ParesBusquedas/servlets/Control_servlet?accion=2\&txt_id_fondo $=2622567$

8 Folleto, «Centro de investigaciones y formación feminista», Madrid, Fondo ANC1-929, Subcarpeta 400, UI. Número 101, Archivo Nacional de Cataluña.

9 Como lo señala Francisco Arriero en su investigación doctoral sobre el Movimiento Democrático de Mujeres, otro de los objetivos del CIFFE fue la elaboración de un libro sobre la historia del feminismo español durante los últimos años del franquismo y la transición. Las entrevistas que realizaron formaban parte de este proyecto, que al final no llegó a publicarse (Arriero, 2015, 48).
} 
del Movimiento Feminista, realizadas por las responsables del centro, Rosa Pardo, Mercedes Comabella, Mercedes Bachiller y Enriqueta Bañón, entre 1985 y 1987, recorriendo la mayor parte de España y realizando centenares de horas de grabación (Arriero, 2015, 47-52).

Más sorprendente y triste es la suerte deparada a los fondos de la Biblioteca de Mujeres de Madrid creada en 1985. Situada en la emblemática calle Barquillo 44 -sede del Movimiento Feminista-, el proyecto surge por iniciativa personal de Marisa Mediavilla Herreros, bibliotecaria y documentalista ${ }^{10}$, a la que se suma un año después la filóloga Lola Robles. Gracias a la voluntad y al tesón de su principal promotora, la Biblioteca reúne en la actualidad unos 30000 ejemplares, entre los que se encuentran libros, revistas, fanzines, tebeos para chicas, estudios y ensayos feministas, femeninos y misóginos, biografías, obras literarias, así como folletos, carteles, pegatinas, o sellos ${ }^{11}$.

En 2006, ante la amenaza de desalojo por parte de la Comunidad de Madrid y ante la imposibilidad de encontrar otro espacio, Marisa Mediavilla cedió el fondo bibliográfico de la Asociación Biblioteca de Mujeres al Instituto de la Mujer para evitar su desaparición. Y en la actualidad se encuentra en el Museo del Traje, perteneciente al Ministerio de Educación, Cultura y Deportes ${ }^{12}$.

\section{Los fondos feministas del Archivo Nacional de Catalunya ${ }^{13}$ : el fondo Lidia Falcón y el fondo Partido Feminista-Club Vindicación Feminista}

Para todas las personas que quieran adentrarse en el estudio del Movimiento Feminista en el Estado español, desde los años 70 hasta los albores del siglo XXI, la consulta de los fondos feministas que alberga el Archivo Nacional de Catalunya supone una visita obligada. Su documentación es igualmente de gran valor para aquellas interesadas en la historia reciente de España, en especial la referente a uno de los episodios más sombríos del siglo XX: la larga dictadura de Franco y la represión policial que ejerció sobre miles de personas.

En 2012, la abogada y escritora feminista Lidia Falcón (1935- ) donó sus fondos al Archivo Nacional de Cataluña situado en la localidad de Sant Cugat del Vallès ${ }^{14}$, en la provincia de Barcelona. El ANC alberga los materiales generados en su actividad dentro de los movimientos sociales, como abogada y escritora -con una bibliografía de más de 50 títulos-, y como fundadora de una de las más relevantes revistas feministas del Estado español, Vindicación Feminista (1976-1979), primero, y Poder y Libertad (1980-) después.

El archivo de Lidia Falcón se divide en dos grandes fondos: uno en torno a sus actividades personales y a su despacho de abogacía, y un segundo y más extenso, alrededor del Partido Feminista, fundado en 1979, y del Club Vindicación Feminista.

10 Robles, Lola: «Biblioteca de Mujeres», Madrid, 2001, p. 6, (documento mecanografiado), Fondo Biblioteca de Mujeres, Museo del Traje.

11 Lores, Alexandra: «La Biblioteca de Mujeres de Madrid de la que nadie habla», El País, 22 de marzo de 2017.

12 Dávalos, Carlos: «Joyas feministas poco conocidas», El País, 18 de agosto de 2017.

13 En el Archivo Nacional de Cataluña se encuentra, además, el fondo de la diputada del PSUC y feminista Dolors Calvet, y el fondo de la escritora Montserrat Roig.

14 Arxiu Nacional de Catalunya, «L'Arxiu Nacional de Catalunya conserva a Catalunya els fons del feminisme de Lidia Falcón». http://anc.gencat.cat/es/detall/noticia/Noticia_LidiaFalcon. (consultado el 24/04/2018) 


\subsection{El fondo Lidia Falcón: una genealogía feminista}

Como su nombre indica, el fondo «Lidia Falcón» ${ }^{15}$ recoge todo tipo de materiales relacionados con su actividad profesional, tanto como abogada en su propio bufete y gabinete jurídico para la Mujer, especializado en la defensa de los derechos de las mujeres, de los trabajadores y de los presos políticos, como también documentos de carácter personal y familiar. Licenciada en Derecho, Arte Dramático y Periodismo, y doctora en Filosofía, el fondo también guarda el testigo de su larga formación académica: su paso, por ejemplo, por el Instituto de Teatro de la Diputación de Barcelona, sus estudios superiores en la Facultad de Derecho de la Universidad de Barcelona, o en la escuela oficial de Periodismo, donde Lidia Falcón se licenció sin poder obtener el título después de una tesina obligatoria ${ }^{16}$.

La ajetreada vida entre Barcelona y Madrid, y la multiplicidad de proyectos en los que se embarcó, llevaron a la abogada a residir en diversos lugares. A este respecto, el fondo recoge documentos sobre su patrimonio, en especial las compra-ventas de sus múltiples residencias, como el inmueble de la calle Roger de Flor 96 de Barcelona, donde Falcón tenía su despacho de abogacía y en donde se elaboró, en los primeros meses, la revista Vindicación Feminista (Falcón, 2012, 112). A través de los documentos oficiales, se puede además reconstruir la «crónica sentimental» de varias generaciones de mujeres que, en una país donde el divorcio se había anulado, las colocaba en un lugar de indefensión total en caso de separación. Casada y madre apenas cumplida la mayoría de edad y separada con tan sólo veinte años, Lidia Falcón vivió en sus propias carnes las dificultades de ser una mujer separada y con hijos en la España de los años cincuenta. El fondo recoge interesante información judicial y penitenciaria como, por ejemplo, la causa de nulidad del matrimonio con su primera pareja, Alfredo Borrás, con el que tuvo sus dos hijos ${ }^{17}$, o la solicitud de matrimonio civil con Eliseo Bayo, periodista y escritor y pareja de la abogada durante veinte años. También se encuentra la adopción por Eliseo Bayo Poblador de Regina y Carlos Enrique Borrás, así como documentación personal y profesional del escritor.

El fondo «Lidia Falcón» no sólo sirve a las/os investigadora/es para reconstruir y estudiar las múltiples vidas de la abogada y escritora, sino también para reconstruir toda la genealogía de su familia, en especial la de las mujeres que la compusieron: Enriqueta O'Neill, la madre de Lidia; Carlota O'Neill, su tía; y Regina del Lamo Jiménez, la abuela de Lidia Falcón, anarquista y escritora, y la primera mujer en introducir el cooperativismo en España ${ }^{18}$.

Destaca la documentación laboral y política de la madre de Falcón, Enriqueta O'Neill, en la Delegación de Prensa y Propaganda del Ministerio de Educación Nacional en Barcelona, donde empieza a trabajar nada más llegar a Barcelona en el año 1941 con toda su familia. De entre los documentos, se incluyen actuaciones de censura de prensa, libros, cine y teatro, así como información sobre la corresponden-

Fondo ANC1-928.

16 Este fue el motivo por el cual Carmen Alcalde se puso a la cabeza de la dirección de la revista Vindicación Feminista, con Marisa Híjar como subdirectora, pues era necesario disponer de una subdirectora colegiada.

17 Para un estudio sobre este periodo de su vida, véase: Falcón, Lidia (2003). La vida arrebatada. Madrid: Anagrama.

18 Regina del Lamo fue fundadora del primer Banco Obrero, en Valencia, en 1920, y fundadora de la Editorial Cooperativa Obrera. «Genealogía», Entrada Regina Lamo, blog sobre Lidia Falcón. http://lafeministalf. blogspot.fr/p/genealogia.html 
cia con las autoridades alemanas e italianas en Barcelona durante la Segunda Guerra Mundial. Como escritora, Enriqueta O'Neill colaboró en numerosos medios, como Siluetas, Moda o Lecturas; también llevó la revista Liceo, del Gran Teatro del Liceo, durante años, y fue constante colaboradora de La Vanguardia ${ }^{19}$. En el fondo se puede encontrar una buena muestra de sus artículos, publicados entre 1942 y 1969, entre los que se incluyen algunos para la prensa venezolana, como Regina Flavio -pseudónimo que adoptó durante la dictadura para poder seguir escribiendo-, así como correspondencia, escritos y recortes de prensa de Venezuela de los años sesenta.

Suerte pareja corrió su tía Carlota O'Neill, quien después de la guerra tuvo que firmar con el pseudónimo de Laura de Noves. Nacida el 27 de marzo de 1925, la tía de Falcón creció en un ambiente de intelectuales progresistas y manifestó un don precoz por la escritura. Prolífica escritora, desde la proclamación de la Segunda República Carlota O'Neill se comprometió con la causa republicana, en especial con la defensa de los obreros a través del Teatro Proletario, pero, sobre todo, con la causa femenina. El 10 de noviembre de 1931 apareció el primer número de la revista Nosotras, que funda con el objetivo de «despertar en la conciencia española, un amplio sentido en política, problemas sociales y económicos» (Allison, 2011, 48). A los pocos días de la sublevación militar, el 22 de julio de 1936, Carlota O’Neill fue encarcelada y pasó cuatro años en la prisión de Melilla, hasta que en 1940 consiguió la libertad condicional ${ }^{20}$. En 1949, asfixiada por la paupérrima posguerra española, Carlota O'Neill decidió exiliarse a Venezuela, con sus dos hijas, Mariella y Lotty, para más tarde ir a México, donde consiguió consagrarse como escritora. En Venezuela, con muy pocos medios, Carlota logra hacerse un hueco en el mundo cultural venezolano, trabajando como periodista, en la televisión, pero sobre todo para la radio. En las cartas, de las que el fondo guarda una cantidad considerable, Carlota describe a su hermana Enriqueta y a «Lily» -apodo cariñoso de Carlota a su sobrina Lidia- su nueva vida en una América «donde reinan las mujeres», su imparable ritmo de trabajo, «tengo que hacer un programa de radio, y otro, y otro» que se va viendo paulatinamente recompensado con el reconocimiento y el confort material desconocido para ellas en la Barcelona de los años cuarenta y que «[han] conseguido con un duro esfuerzo» ${ }^{21}$.

Periodista y escritora, con grandes dotes para el canto y la música, en especial para el piano, la abuela de Lidia Falcón, Regina del Lamo Jiménez, es la primera de las mujeres de esta genealogía que despunta desde las postrimerías del siglo XIX por su activismo en la difusión de las ideas del sindicalismo y el cooperativismo, escribiendo sobre un sinfín de temas como el control de la natalidad, el aborto o el amor libre, pero también «artículos, proclamas, manifiestos que debían inflamar la conciencia del proletariado revolucionario [...] codo con codo con el líder catalán más destacado de aquellos tiempos, Lluís Companys» (Falcón, 1989, 32). Este fondo conserva, además de documentos personales como, por ejemplo, su certificado de matrimonio con Enrique O'Neill -de ascendencia nobiliaria y quince años mayor que Regina-, textos políticos, ensayos, libros, notas y borradores de textos y poemas

19 Entrevista a Lidia Falcón. Periodistes en temps dificils http://periodistesentempsdificils.adpc.cat/?p=175; (Consultado el 02/05/2018)

20 Carlota O’Neill cuenta su experiencia en la cárcel en su libro de memorias (O’Neill, 2003, 244-245).

21 Carta de Carlota O'Neill, 29 de diciembre (s.f.) a Enriqueta O'Neill. Fondo ANC1-928, Carpeta 1673, UI. Número 298, Archivo Nacional de Cataluña. 
de casi tres décadas (1923-1944), y los primeros textos sobre cooperativismo, escritos entre los años 1923-1927.

Aunque muy breve, en el fondo Lidia Falcón se incluye también documentación sobre su padre, César Falcón (1892-1979), creador del Teatro Proletario, periodista y fundador del Partido Socialista en Perú además de director del Mundo Obrero (Mangini, 2001, 196-198).

\subsection{El fondo Partido Feminista de España - Club Vindicación Feminista ${ }^{22}$ : la historia de una larga lucha}

Si el fondo Lidia Falcón recorre la documentación generada principalmente en su actividad laboral como abogada en su gabinete jurídico y psicológico para la mujer, así como la documentación destacada referente a las mujeres de la familia LamoO’Neill, el segundo fondo gira entorno a los materiales generados por el Partido Feminista de España (PFE) y el Club Vindicación Feminista desde los años 70 hasta el inicio de los años 2000. En cuanto a su catalogación, el fondo está dividido en diez series $^{23}$ que recogen toda la documentación del PFE, pasando por la labor editorial del Club Vindicación Feminista y sus sociedades editoras, Ediciones de Feminismo, S.A., creada en 1976, y su sucesora Vindicación Feminista Publicaciones, fundada en 1979, la documentación generada por las revistas Vindicación Feminista, y más tarde Poder y Libertad, hasta la documentación sobre el centro de información y recursos y la rica hemeroteca del partido.

Sucesor de la Organización Feminista Revolucionaria ${ }^{24}$, el Partido Feminista de España se constituye en mayo de 1979, tras dos años de reflexión sobre «el tema de la mujer como clase social explotada y oprimida por el hombre», llegando «a las mismas conclusiones que otros grupos de mujeres de diversos países del mundo: la mujer es una clase social y económica» ${ }^{25}$, pero no se legaliza, tras superar dos años de arduas trabas burocráticas, hasta el 8 de marzo de 1981.

Dentro de la documentación producida y recibida por el PFE se encuentra la documentación constitutiva (acta de constitución en la notaría de Ignacio Zabala de Barcelona, estatutos desde 1980 hasta 1999), actas de reuniones de la comisión ejecutiva, congresos del Partido Feminista, fichas de las socias o correspondencia con militantes del partido. Es de destacar el listado de direcciones y contactos con otros partidos feministas. Y es que, desde su publicación en 1979, las tesis ideológicas del PFE recogidas en el libro Partido Feminista. Tesis, se convierten en un texto de referencia a nivel europeo que sirven de base teórica para la constitución de partidos homólogos extranjeros. Por ejemplo, el cinco de marzo de 1980, recién cumplido el

22 Fondo ANC1-929

23 Para ver el inventario del fondo: http://anc.gencat.cat/web/.content/anc/documents/arxiu/Quadre-del-Fons-Partido-Feminista-de-Espana-Club-Vindicacion-Feminista.pdf

24 La Organización Feminista Revolucionaria se creó en 1977 tras la expulsión, en abril del mismo año, de Lidia Falcón, Regina Bayo y Anna Estany del Colectivo Feminista de Barcelona. El Colectivo Feminista de Barcelona se había creado en el verano de 1975 por Lidia Falcón y María José Ragué Arias, tras el germen de una reunión en febrero de 1974 en la que participaron «10 mujeres entre las que se encontraban Adela Tomás, Regina Bayo, Lidia Falcón, Carmen Alcalde, María José Ragué Arias y Ana Estany», Colectivo Feminista de Barcelona, «Comunicado de colectivo feminista de Barcelona», p. 1, (s.f.), (texto mecanografiado), Fondo ANC1-929, Caja UC 31, Subcarpeta 6, Archivo Nacional de Cataluña.

25 «La necesidad de los Partidos feministas» p. 1, (s.f.), (documento mecanografiado), Fondo ANC1-929, Caja UC 27, UC. Número 6, Archivo Nacional de Cataluña. 
primer aniversario del PFE, Lidia Falcón recibe una carta de Rosmarie Brupbacher, que ha fundado en Basilea un grupo feminista con pretensión de constituirse en partido. En su carta, la feminista suiza cuenta cómo ha sabido de la existencia del PFE: Gunild Feigenwinter, feminista alemana y fundadora de una editorial feminista en Basilea, le había pedido la traducción al alemán de las tesis del Partido Feminista de España, y ahora escribe interesada en tomar esas tesis como corpus teórico para su partido y para otro en Alemania, donde también milita ${ }^{26}$.

Si la actividad política en torno al PFE es uno de los polos del segundo fondo, la labor periodística y editorial constituye el otro pilar de su documentación.

Fundada en julio de 1976 por Lidia Falcón y Carmen Alcalde, la revista Vindicación Feminista fue sin duda el mayor exponente de la prensa feminista en los años 70 , tanto por su tirada -en algunos meses se llegaron a alcanzar los 35.00 ejemplares, aunque la media se situaba habitualmente en unos 15000 (Larumbe, 2004, 173), como por su calidad gráfica y su contenido, analizando con ironía y profundidad todos los temas que incumbían a las mujeres, sin dejar de lado una mirada sarcástica y crítica sobre la actualidad política del país y las vicisitudes que estaban acaeciendo. Desde las páginas de Vindicación se advirtió, por ejemplo, de las derivas reformistas que estaban produciendo en la política y del riesgo que corrían todos los españoles, pero en especial las mujeres, de servir de moneda de cambio para los pactos y las concesiones ${ }^{27}$. Vindicación Feminista era también el elenco de escritoras e intelectuales de renombre que colaboraron de forma más o menos regular en las páginas de la publicación, como Montserrat Roig, Ana Moix, Colita, Juana Gallego, Marta Pessarrodona, Maruja Torres o Antonina Rodrigo, por citar solo algunas.

En la serie número siete del fondo, además de la documentación sobre Vindicación Feminista, se encuentra toda la documentación generada por la revista Poder y Libertad, fundada en 1979 como órgano de expresión del Partido Feminista de España. Sobresale, además, la intensa labor editora que emprendió el grupo fundador de Vindicación Feminista, y del Club Feminista después, a través de sus proyectos editoriales. El fondo guarda el testimonio de esta ingente labor editorial: obras publicadas, traducciones de obras extranjeras -en el archivo se encuentra, por ejemplo, el contrato para la publicación de la traducción de la obra El rapto de los orígenes $o$ el asesinato de la madre, de Suzanne Blaise, que se tradujo al castellano en 1996- o información sobre iniciativas editoriales como la creación, en 1984, de la colección de narrativa «Escritura de mujer», entre la Editorial Hacer y el Club Vindicación Feminista, que buscaba «acoger todas las producciones de las autoras que crean en el arma que es la literatura y la utilicen al servicio de sus hermanas de sufrimiento» ${ }^{28}$.

El 3 de noviembre de 1981 se inaugura en Barcelona el Club Vindicación Feminista como «Asociación para la Asistencia e Información a la mujer, así como centro

26 Carta de Rosmarie Brupbacher a Lidia Falcón, 5 de marzo de 1980, Fondo ANC1-929, Carpeta 2161, Subcarpeta 394, Archivo Nacional de Cataluña.

27 Nos referimos en concreto a la despenalización del aborto y a la legalización del divorcio, asuntos que se dejaron de lado en la Constitución de 1978. Una crítica a la Constitución la encontramos en varios artículos de la revista. Véase: Oranich, Magda: «La Constitución: piedra de toque», Vindicación Feminista, nº 22, abril 1978, p. 21; Oranich, Magda : «La vieja historia de siempre. La Constitución: Oprimidas como siempre», Vindicación Feminista, n 26-27, septiembre 1978, p. 8; Falcón, Lidia: «La Constitución: Las Españolas ni fu ni fa. El pene sigue siendo el rey», Vindicación Feminista, nº 25, julio 1978, pp. 17-18.

28 Sin título (documento mecanografiado) 31 de mayo de 1984, p. 5, Fondo ANC1-929, Carpeta Código 31, Subcarpeta UI. Número 6, Archivo Nacional de Cataluña. 
de reunión, discusión, diversión y estudio» ${ }^{29}$. En un primer momento, el Club se instala en la calle Bailén 18 de Barcelona y se convierte rápidamente en un espacio multifuncional como «sala de conferencias pero también como pequeño bar», hasta que en 1986 Lidia Falcón y Elvira Siurana se trasladan a Madrid y deciden utilizar de campamento base el primer piso del número veintinueve de la calle Magdalena de Madrid (Falcón, 2012, 285). Desde mediados de los años ochenta, el Club Vindicación Feminista impulsó, además, una enorme labor de asistencia y servicios para las mujeres y sus hijos «con un equipo de abogadas, psicólogas, sexólogas, trabajadoras familiares, asistentes sociales y ginecólogas $»{ }^{30}$. La documentación sobre el Club Vindicación se extiende desde el año de su creación hasta el año 2009, y recogen toda clase de documentos sobre su constitución, su gestión y sus múltiples actividades.

\subsection{Correspondencia, colaboraciones y amistad}

Restablecerlos los vínculos entre las feministas españolas y las homólogas extranjeras es otra de las múltiples posibilidades que brinda el archivo a quienes quieran adentrarse en sus legajos. En cuanto a Vindicación, el proyecto feminista de la revista se inscribe claramente en una línea internacionalista que entronca con los eslóganes internacionales del momento, Women of the world, unite!, que parte de una concepción marxista de la mujer como clase económica oprimida -a la que se añadirá el componente de la opresión sexual-reproductiva-. Vindicación aspira a convertirse no solo en el portavoz de los feminismos del Estado español, sino también en plataforma de contacto entre organizaciones y colectivos de diversas nacionalidades. Secciones como «Mujeres del Mundo», «Internacional» o entrevistas a reconocidas activistas internacionales, tenían como objetivo informar de lo que sucedía más allá de las fronteras, así como poner en contacto grupos y colectivos. El componente internacionalista no es solo patente en el contenido de la revista, sino también en las propias relaciones que mantuvo el equipo editorial con otros colectivos y revistas homólogas extrajeras. De hecho, en el fondo Partido Feminista-Club Vindicación Feminista se puede reconstruir una parte importante de la correspondencia entre las colaboradoras de Vindicación Feminista primero, y las militantes del Partido Feminista, después, con otros colectivos extranjeros, así como la suscripción de numerosas librerías y universidades extranjeras a la publicación.

A este respecto, numerosas homólogas extranjeras escriben a Vindicación para solicitar información sobre la misma, para colaborar o, incluso, para ayudarse económicamente. Hildegard Kawan, fundadora de la revista feminista alemana Courage, editada en Berlín, escribe, por ejemplo, a Vindicación Feminista en el año 1979 para proponerle un intercambio de revistas y una posible colaboración, señalando que, como colaboradora de una revista feminista, «tenemos interés en las [sic] problemas feministas españolas también ${ }^{31}$. Los problemas financieros que atraviesa Vindicación llegan a oídos de otras publicaciones extranjeras. El mismo año, Jill Nichols, colaboradora de

29 Folleto presentación «Club-Vindicación Feminista», 1981, Fondo ANC1-929, Caja Código 400, Subcarpeta UI. Número 101, Archivo Nacional de Cataluña.

30 Carta de Montserrat Fernández dirigida al Instituto de la Mujer, julio de 1984, p. 2, Fondo ANC1-929, Caja Código 57, Subcarpeta UI. Número 10, Archivo Nacional de Cataluña.

31 Carta de Hildegard Kawan a Vindicación Feminista, 10 de septiembre de 1979, Fondo ANC1-929, Carpeta Número. 394, Subcarpeta 2159-216, Archivo Nacional de Cataluña. 
la revista inglesa Spare Rib ${ }^{32}$ escribe al equipo de Vindicación informando de que se ha enterado de los problemas financieros que atraviesa la revista catalana que le impiden seguir publicándose -al igual que la revista italiana $E f f e^{33}$, como también evoca de pasada Jill Nichols, de la que el fondo del Partido Feminista-Club Vindicación conserva casi todos los números. Al final de la carta, Jill Nichols pide al equipo de Vindicación que le remita un texto que explique las dificultades por las que atraviesa la revista para intentar crear un fondo de donaciones «si fuera necesario» ${ }^{34}$.

Otras veces las cartas son individuales, de lectoras que viven en el extranjero, muchas de origen español, que escriben a la revista para expresar su opinión sobre la misma, solicitar algún número o hacer sugerencias. Así, por ejemplo, en un español algo inseguro, escribe Petra Hartmann, de Frankfurt, con fecha del 22 de mayo de 1979. Por su carta sabemos que tienen veintiún años, que es estudiante de sociología y que ha descubierto la dirección de Vindicación Feminista por la revista Courage, a la que hacía referencia Hildegard Kawan. Desea ir a España en verano para conocer mejor el país, entrar en contacto con grupos feministas españoles y mejorar su nivel de español. Pide, por favor «que vosotros imprimáis mi carta en vuestra revista, para que estas personas puedan escribirme ${ }^{35}$. Otras veces se trata de cartas de organismos, de universidades o de bibliotecas que desean suscribirse a la revista o hacer intercambios. Si bien es difícil poder rastrear la continuación de estas relaciones epistolares, los testimonios que nos quedan revelan, por un lado, la difusión internacional de Vindicación, lo que facilitó, sin duda, que se dieran a conocer las luchas feministas en España y los colectivos que participaban en ellas y, por otro, la existencia de una activa circulación de ideas, reivindicaciones y textos entre colectivos feministas que, en distintas partes del mundo y en contextos sociopolíticos y culturales diferentes, estaban gestando reflexiones análogas.

De entre la correspondencia, una gran parte se entabló con el país vecino. Podemos destacar la relación de amistad entre Lidia Falcón y la feminista francesa Antoinette Fouque, así como la relación de colaboración de las dos editoriales, a saber, Vindicación Feminista Publicaciones y el proyecto editorial Éditions des femmes creado por la propia Fouque. De hecho, las trayectorias de la feminista francesa y de Falcón guardan grandes parecidos. Y es que ambas tuvieron un papel preponderante en el germen del Movimiento feminista en sus respetivos países. Psicoanalista de formación, en plena resaca de mayo del 68 Antoinette Fouque cofunda el Mouvement des Libération des femmes (MLF) en octubre de 1968 y crea la tendencia conocida como Psicoanálisis y Política, que da nombre a su grupo Psy et po (Fouque, 2009). A finales de 1973, funda y dirige las Éditions des femmes, la primera editorial en Europa dedicada a la creación de las mujeres, y enseguida inaugura las librerías des femmes en 1974, 1976 y 1977 (Cresson et al., 251), siendo la más mítica la de la calle Jacob, en Paris, que continúa en la actualidad.

32 Spare Rib fue una de las primeras revistas feministas. Se publicó entre 1972 y 1993. Tanto por la orientación feminista como por el diseño guarda un gran parecido con Vindicación Feminista. Para ampliar información sobre esta publicación inglesa, véase: https://journalarchives.jisc.ac.uk/britishlibrary/spareri

33 Effe fue una revista feminista italiana fundada en 1973. Se caracterizaba por su diseño cuidado y su gran calidad fotográfica. En 1981 cerró por una deuda acumulada de 5 millones de liras (Gallego, 1990, 178).

34 Carta de Jill Nicholls a Vindicación Feminista, 25 de octubre de 1979, Fondo ACN1-929, Carpeta, Número 394, Subcarpeta 2159-2161, Archivo Nacional de Cataluña.

35 Carta de Petra Hartmann a Vindicación Feminista, 25 de mayo de 1979, Fondo ANC1-929, Carpeta 394, Subcarpeta 2159-2161, Archivo Nacional de Cataluña. 
La relación con la feminista gala remonta a finales del año 1974, cuando la abogada, junto con sus hijos, y Eliseo Bayo, su compañero del momento, es detenida por la Brigada Político Social el lunes 16 de septiembre de 1974, acusados todos de haber participado en el atentado perpetrado por ETA de la calle Correo de Madrid $^{36}$. A partir de ese momento, Antoinette Fouque y las compañeras de Des Femmes comienzan una larga campaña de apoyo y de movilización para liberar a los encarcelados que las llevó a publicar, por ejemplo, en Le Monde, un extenso manifiesto a favor de los detenidos y contra la represión franquista firmado por 505 mujeres, entre las que se incluyeron personalidades como las escritoras Françoise d'Eaubonne, Françoise Sagan o la feminista norteamericana Kate Millett ${ }^{37}$.

Antoinette Fouque y la editorial Des femmes son, además, las promotoras de editar al francés el libro de Falcón Cartas a una idiota española, publicado el mismo año de su detención por la editorial Dirosa, que el grupo de Antoinette Fouque descubre en la Feria del libro de Fráncfort en 1974. Tras la detención, varias militantes de Des femmes van a Barcelona para adquirir los derechos de su libro en Francia (Fouque, $2004,104)^{38}$, cuyo prólogo escribe Lidia Falcón desde la cárcel ${ }^{39}$. Dos años más tarde, Antonitte Fouque y la editorial Des femmes publican también la traducción al francés del libro de Falcón En el infierno. Ser mujer en las cárceles de España, escrito durante la estancia en Yeserías, donde retrata la experiencia en la cárcel y la historia de las mujeres que conoció durante su confinamiento, denunciando el maltrato, el vilipendio y la vejación que sufren las mujeres en las instituciones penitenciarias franquistas.

La segunda francesa con la que Lidia Falcón mantiene una nutrida relación epistolar, que se encuentra en el Archivo Nacional de Catalunya, es Suzanne Blaise, creadora del Partido Feminista Unificado (PFU) en Francia en octubre de $1975^{40}$. El fondo Partido Feminista-Club Vindicación Feminista se halla, además de un nutrido intercambio epistolar entre las dos feministas, una numerosa documentación sobre el Partido Feminista galo (actas, textos teóricos, textos de base, presentación del partido) así como sobre el Parti Féministe belge, cuya promotora es igualmente Suzanne Blaise. De hecho, los dos partidos son los promotores del intento de creación de una Internacional Feminista, que se impulsa en Paris en mayo de 1977, cuyo objetivo era crear «una solidaridad efectiva y contactos regulares entre mujeres de todos los países $\rangle^{41}$ y al que

36 Para saber más sobre la detención, véanse las memorias de la propia Falcón, Lidia (1981). Viernes y 13 en la Calle del Correo. Barcelona: Planeta.

37 «Lutte et libération des femmes espagnoles», Le Monde, 31 octubre 1974, Fondo ANC1-928, Carpeta 1436, UI. Número 257, Archivo Nacional de Cataluña.

38 Según Lidia Falcón, una parte de los beneficios de la edición francesa del libro servirían para sufragar gastos para la fianza. Entrevista personal a Lidia Falcón, 22 de marzo de 2018.

39 En el prólogo de la edición francesa, Lidia Falcón cuenta de dónde vino la idea de escribir el libro. Las cartas tenían como origen contestar a uno de los best-seller de la época, El varón domado, de Esther Vilar. Estas cartas se publicaron semanalmente en el diario Sábado Gráfico, dirigido por Eugenio Suárez, desde 1973. El libro era una recopilación de 10 cartas junto a otras 10 inéditas escritas para la ocasión. Las ilustraciones corrieron a cargo de Nuria Pompeia, Falcón, Lidia (1975). Lettres à une idiote espagnole. Paris: Des femmes, pp. 15-16.

40 El Partido Feminista unificado (PFU) fue cofundado el uno de octubre de 1975 por Suzanne Blaise-Rigail, después de su escisión del Parti féministe (1974-1978). El objetivo del PFU era doble : la «lutte contre le patriarcat, lutte contre le capitalisme, et le choix de société: une société sans classes (donc autogestionnaire) d'où soit exclue toute oppression», Informe « France : Parti Féministe Unifié », p. 1, (sf), (texto mecanografiado), Fondo ANC1-929, Carpeta 74, Archivo Nacional de Cataluña.

41 « Nous prendrons contact très prochainement avec le PF allemand et tous les partis féministes intéressés pour jeter les bases d'une Internationale de ces Partis dont le but serait une solidarité effective et des contacts réguliers entre femmes des tous les pays », Carta de Suzanne Blaise a Lidia Falcón, Paris 22 de enero de 1977, Fondo ANC1-929, Carpeta 381, Subcarpeta UI. Número 90, Archivo Nacional de Cataluña. 
invitan a participar al Colectivo Feminista de Barcelona. Aún en Europa, destaca también la relación de la abogada con Gerd Brantenberg, militante feminista y escritora noruega, y con Gunild Feigenwintery, militante alemana, creadora de una de las revistas feministas más relevantes de los setenta, Die Hexenpresse, y una de las promotoras de la traducción al alemán de las tesis del Partido Feminista de España.

Si bien Francia aparece como agente privilegiado de los primeros intercambios -aunque la amistad con Antoinette Fouque o Suzanne Blaise prosigue muchos años más-, a partir de la década de los 80 se intensifican las relaciones con las feministas norteamericanas. Lidia Falcón aterriza por primera vez en Estados Unidos en 1982, pero la relación con las norteamericanas se remonta a 1974, cuando, en pleno bullicio de la elaboración de La razón feminista, pocos meses antes de ser detenida, Gloria Waldman y Linda Levine visitan a Lidia Falcón para realizarle una entrevista destinada al proyecto que se traen entre manos sobre el feminismo en el franquis$\mathrm{mo}^{42}$. De su primera visita a Estados Unidos arranca la amistad de Falcón con un nutrido grupo de feministas norteamericanas, la mayoría profesoras e investigadoras en diferentes universidades estadounidenses, entre las que se encuentran las anteriormente citadas Gloria F. Waldman, de la Universidad de New York, y Linda G. Levine, de la Universidad de Montclair, Marie Lise Gazarian, de la Universidad de St. John o Patricia O'Connor, de la Universidad de Cincinnati, la gran difusora del «teatro feminista español» en Estados Unidos (Falcón, 2012, 245).

Otro de los ambiciosos proyectos en los que se embarcó Falcón con sus homólogas norteamericanas en la década de los ochenta, y que se puede reconstituir en detalle en los fondos del ANC, fue Sisterhood is Global, una enciclopedia internacional sobre la situación de las mujeres en ochenta países del mundo que da nombre a una fundación homónima (Falcón, 2012, 250-252), cuyo objetivo era la creación de una red de colaboración y alianzas entre colectivos y partidos feministas de todo el mundo que funcionaría como el «primer think-tank feminista internacional (...) para examinar la política global desde la perspectiva de las mujeres» ${ }^{43}$. La propuesta parte de Robin Morgan, que en esta época es, además, subdirectora de la famosa revista feminista norteamericana $M s$, fundada y dirigida por Gloria Steinem en colaboración con Karen Berry, quien se pone a la cabeza del Sisterhood Is Global Institute. Este proyecto es el arranque de contactos y de otras iniciativas que se mantienen en el tiempo y que requerirán gran parte de los esfuerzos de Lidia Falcón y, en menor medida, del PFE, y que revelan una vez más la marcada dimensión internacionalista que recorre toda la trayectoria militante de la escritora y abogada.

42 Su proyecto se materializó unos años después en el libro: Levine, Linda. G.,y Waldman, Gloria (1980). Feminismo ante el franquismo: Entrevistas con feministas de España. Miami: Ediciones Universal.

Tras su detención, ambas participaron activamente en la campaña por la liberación de Lidia Falcón tras el atentado de la calle del Correo, y consiguieron que la escritora Barbara Probst Salomon publicara un artículo en The New York Times hablando sobre la tortura en España.

Salomon Probst, Barbara, «Torture in Spain»: The New York Times, 25 de noviembre de 1974.

https://www.nytimes.com/1974/11/25/archives/torture-in-spain.html

43 "This is a historic occasion - the first international feminist "think-tank" ever to assemble, to examine global politics from perspective of women», Carta de Simone de Beauvoir enviada al I encuentro de Sisterhood is Global, 19 de noviembre de 1984, Fondo ACN1-928, Carpeta 1692, Archivo Nacional de Cataluña. 


\section{Conclusiones}

Las cinco vidas de Lidia Falcón ${ }^{44}$ reza el título de un reciente libro homenaje a su figura. Y es que, de hecho, como hemos intentado mostrar, son varias las facetas que se pueden estudiar a través de sus fondos, en los múltiples campos en los que desplegó sus competencias y ejerció su lucha como abogada, periodista, escritora, dramaturga y militante feminista, junto con otras mujeres y colectivos que le han acompañado a lo largo de su dilatada trayectoria y con las que mantuvo, en muchos casos, una nutrida relación, como muestran las cartas que se han podido conservar.

Dentro de los fondos, destaca la documentación generada tanto en su militancia feminista en distintos grupos como el Colectivo Feminista de Barcelona, primero, y la Organización Feminista Revolucionaria después, como en su labor periodística y de editora de una de las revistas capitales de los años setenta, Vindicación Feminista. A través de estos materiales se pueden reconstruir, por ejemplo, las distintas actividades y campañas llevadas a cabo por el Movimiento feminista que dan cuenta del dinamismo y la diversidad de las organizaciones feministas en una de las etapas claves de la historia reciente de España.

Si bien en el Estado español no contamos con un Centro del Feminismo -un archivo que reuniese toda la memoria feminista de manera centralizada-, los fondos feministas del Archivo Nacional de Catalunya, del que solo hemos comentado una parte, son una iniciativa capital de conservación y catalogación de parte de esa memoria feminista, que se va poco a poco completando con otras iniciativas ${ }^{45}$ y que facilitan una aproximación enormemente completa a la historia del feminismo del Estado español durante las últimas décadas del siglo XX. Además, aunque en este texto no se ha abordado, el fondo de Lidia Falcón y del Partido Feminista-Club Vindicación no solo recogen documentos generados en su ejercicio. En este sentido, destaca la ingente hemeroteca del Partido Feminista, ya que tanto Lidia Falcón como el PFE funcionaron como coleccionistas de facto, conservando ingentes cantidades de literatura gris, en especial fanzines y revista de muy pequeña tirada, generada por otros grupos y colectivos feministas nacionales e internacionales. Documentación que sirve también para rastrear las huellas de otros grupos o publicaciones que tuvieron una vida más efímera y que, por tanto, están más expuestos a una memoria feminista más vulnerable.

Fondos feministas como los del Archivo Nacional de Cataluña son, pues, fundamentales para reconstruir y transmitir las memorias del feminismo y para contrarrestar la destrucción y el olvido a las que están expuestas, inevitablemente, las memorias calientes.

\section{Agradecimientos}

La autora quiere agradecer la lectura y las precisiones del texto a Marisa Mediavilla, de la Biblioteca de Mujeres de Madrid, y a Lidia Falcón por la autorización de la consulta de los fondos de acceso restringido del Fondo ANC1-929.

44 Tomamos prestado el título del libro homenaje a Lidia Falcón. (Camps et al., 2015)

45 Tenemos que destacar, por ejemplo, la labor de digitalización de una enorme cantidad de material de grupos feministas llevada a cabo por el Centro de documentación de Emakumeak. http://cdd.emakumeak.org/recursos/ lista_documentos 


\section{Referencias bibliográficas}

Alcalde, Carmen (1976). La mujer en la Guerra Civil española. Madrid: Cambio 16.

- (1996). Las mujeres en el Franquismo. Barcelona: Flor del Viento Ediciones.

Almerini, Katia (2014). LaSal, bar-biblioteca feminista en Barcelona. Empoderamiento femenino y cultura visual. Boletín de Arte, 35, Departamento de Historia del Arte, Universidad de Málaga, 83-100.

Arriero, Francisco (2015). El Movimiento Democrático de Mujeres, del antifranquismo a la movilización vecinal y feminista. Ideología, identidad y conflictos de género, Tesis Doctoral, Universidad Autónoma de Madrid, Madrid.

Arxiu Nacional de Catalunya «L'Arxiu Nacional de Catalunya conserva a Catalunya els fons del feminisme de Lidia Falcón», http://anc.gencat.cat/ca/detall/article/Noticia_LidiaFalcon (consultado el 02/01/2018)

Bard, Christine et al. (2006). Guide des sources de l'histoire du féminisme. Rennes: Presses Universitaires de Rennes.

Cervera, Montse et al. (1997). Ca la dona. Barcelona: Fundación Serveis de Cultura Popular.

Codina-Canet, María Adelina y San Segundo, Rosa (2016). Propuesta de un Centro de Archivo del Feminismo tras el análisis de los fondos documentales del Movimiento Feminista. Revista Española de Documentación Científica, 39 (1), 1-14. doi: http://dx.doi. org/10.3989/redc.2016.1.1245

Cresson, Edith et al. (2014). Avec Antoinette Fouque hommage. Paris : Des femmes-Antoinette Fouque.

Falcón, Lidia (1989). Los hijos de los vencidos. Madrid: Vindicación Feminista Publicaciones.

- (1999). Memorias políticas (1959-1999). Madrid: Vindicación Feminista Publicaciones.

- (2012). La pasión feminista de mi vida. Barcelona: El Viejo Topo.

Jornet i Benito, Núria y Tuset Páez (2016). Construyendo la memoria de los feminismos: archivos, bibliotecas y centros de documentación. Una mirada al pasado, una reflexión para el futuro", BiD: textos universitaris de biblioteconomia i documentació, 36. doi: http:// dx.doi.org/10.1344/BiD2016.36.10

Llinàs Carmona, Conxa (2008). Feminismes de la Transició a Catalunya. Textos i materials. Barcelona: Horsori Editorial.

Mangini, Shirley (2001). Las modernas de Madrid. Las grandes intelectuales españolas de la vanguardia. Barcelona: Ediciones Península.

Mediavilla, Marisa y Robles, Lola (2000). Las librerías de mujeres en España. Métodos de Información, 7 (35-36), 57-61.

O’Neill, Carlota (2003). Una mujer en la guerra de España. Madrid: Oberon.

Rich, Adrienne (2011). Sobre mentiras, secretos y silencios. Madrid: Horas y Horas.

Rosón, María y Medina Domenech, Rosa (2017). Resistencias emocionales. Espacios y presencias de lo íntimo en el archivo histórico. Arenal, 24 (2), 407-439.

Taillot, Allison (2011). De Carlota O’Neill à Lidia Falcón : le féminisme de la Transition entre héritage et rupture. En Marie-Claude Chaput (Ed.): Masculin/Féminin en transition, (pp. 47-61), Paris: Université Paris Ouest Nanterre La Défense. 
